\title{
The predictability of T3 disease in staging MRI following prostate biopsy decreases in patients with high initial PSA and Gleason score
}

\begin{abstract}
Young Hwii Ko ${ }^{1}$, Deuk Jae Sung ${ }^{2}$, Sung Gu Kang ${ }^{1}$, Seok Ho Kang ${ }^{1}$, Jeong Gu Lee ${ }^{1}$, Je Jong Kim ${ }^{1}$ and Jun Cheon ${ }^{1}$
To obtain improved accuracy in predicting extracapsular extension (ECE) and seminal vesicle invasion (SVI), we evaluated the variables affecting the predictability of staging magnetic resonance imaging (MRI, phased-array coil) and estimated their impact on accuracy between preoperative MRI staging and histological outcome. A total of 121 patients with localized or locally advanced prostate cancer who underwent robotic radical prostatectomy (RALP) were included. Following transrectal biopsy, all enrolled patients had undergone MRI for staging work-up. After RALP, only 43.8\% (53/121) of the patients were matched with the MRI predicted stage. Compared to the matched group in the prediction of ECE, the unmatched group had significantly higher initial prostate-specific antigen (PSA, $12.8 \mathrm{ng} \mathrm{ml}^{-1}$ versus $\left.8.1 \mathrm{ng} \mathrm{ml}^{-1}, P=0.048\right)$. In the prediction of SVI, initial PSA $\left(8.1 \mathrm{ng} \mathrm{ml}^{-1}\right.$ versus $\left.17.3 \mathrm{ng} \mathrm{ml}^{-1}, P=0.009\right)$ and biopsy Gleason score ( 6.5 versus $7.6, P=0.035)$ were significantly higher in the unmatched group. When applying clinical cutoffs of initial PSA of 10 and $20 \mathrm{ng} \mathrm{ml}^{-1}$, the accuracy of MRI in the prediction of ECE was decreased in the group with PSA over $20 \mathrm{ng} \mathrm{ml}^{-1}$ $(75.6,64.5$ and $37.5 \%, P=0.01)$, and this group had significantly decreased accuracy of MRI in the prediction of SVI $(91.5,77.4$ and $37.5 \%, P<0.01$ ). Applying the clinical cutoff of a Gleason score of 7, the accuracy of MRI in the prediction of SVI was decreased in the higher Gleason score group $(93.9,82.1$ and $62.9 \%, P=0.01)$. Thus, for these patient groups, to obtain margin negativity during radical prostatectomy, operative findings, rather than post-biopsy MRI images, may provide substantial information, implying a clinical advantage in conducting MRI before prostate biopsy.
\end{abstract}

Asian Journal of Andrology (2011) 13, 487-493; doi:10.1038/aja.2010.165; published online 7 March 2011

Keywords: magnetic resonance imaging; neoplasm staging; prostatic neoplasms

\section{INTRODUCTION}

When evaluating prostate cancer prior to decisive procedures, knowledge of the extent of the disease can enable improved oncological control $^{1}$ by changing treatment modality or surgical refinement. Considering that the margin positivity and risk of recurrence are higher in T3 disease with extracapsular extension (ECE) or seminal vesicle invasion (SVI), improved preoperative staging is pivotal. To obtain this, magnetic resonance imaging (MRI), which provides the best depiction of the contours of the prostate as well as its internal zonal anatomy, has emerged as the most promising method for radiological evaluation, allowing evaluation of tumour location, tumour volume, capsular penetration, invasion of the neurovascular bundle and seminal vesicle involvement ${ }^{2-4}$ according to recent reports in the literature.

Although it is routinely used as a preoperative radiological evaluation, there are several limitations associated with local staging of the prostate by MRI after pathological confirmation of prostate cancer via prostate biopsy. Low-signal intensity lesions in the peripheral zone may also be caused by several other factors, including post-biopsy haemorrhage, prostatitis, intraglandular dysplasia and benign prostatic hyperplasia. ${ }^{5-8}$ In particular, haemorrhage after biopsy, which is generally observed during the first 8 weeks after the procedure, is a substantial limitation of MRI in this setting, resulting in both overand underestimation of tumour extent. ${ }^{9,10}$ Moreover, MRI cannot detect microscopic invasion; none of the currently available imaging modalities allows for detection of microscopic invasion. ${ }^{11}$ All of these factors together affect the accuracy of MRI as a staging work-up, resulting in considerable variation in terms of sensitivity and specificity: $14.4-100$ and $67-100 \%$, respectively.

Thus, to obtain improved accuracy in predicting ECE and SVI, we evaluated the variables affecting the predictability of staging MRI and estimated the variables' impact on accuracy between preoperative MRI and pathological outcome. Efforts to identify the variables may not only allow refinements in surgical technique but also improve overall oncological outcomes by providing detailed information on ECE and SVI status, which facilitates obtaining negative margins.

\section{MATERIALS AND METHODS}

Patient selection and data collection

A total of 121 patients with localized or locally advanced prostate cancer (clinical stage T1c to T3c) diagnosed by transrectal prostate biopsy who underwent robotic radical prostatectomy (RALP) from July 2007 to December 2009 in our institution were included in this

${ }^{1}$ Department of Urology, Korea University School of Medicine, Seoul, Korea and ${ }^{2}$ Department of Radiology, Korea University School of Medicine, Seoul, Korea Correspondence: Dr J Cheon (urokyh@gmail.com)

Received: 22 August 2010; Revised: 1 November 2010; Accepted: 16 November 2010; Published online: 7 March 2011 
study. Following transrectal biopsy, all enrolled patients had undergone MRI following the same protocol for staging work-up, mainly for preoperative staging for the purpose of refining the surgical plan. Because of the anxiety of patients who might prefer early surgery rather than a waiting period of $4-8$ weeks after biopsy, as well as recent data suggesting no relationship between time to surgery after transrectal biopsy and operative outcome, our policy for radical prostatectomy is one with a minimal wait after confirmation of histological confirmation of the diagnosis. Thus, we have no previously set period for MRI evaluation after initial biopsy.

RALP procedures were performed by a single experienced full faculty robotic surgeon (J. Cheon). Following prostatectomy, resection specimens were fixed in $10 \%$ neutral buffered formalin for $36 \mathrm{~h}$. After inking of the margins, routine sections of all of the surgical margins, including the prostatic base, apex and peripheral zone, capsule, periprostatic soft tissue, seminal vesicle, urethra and bladder neck, were examined with permanent staining. Based on these findings, ECE, SVI and positive surgical margins were evaluated by a single full faculty uropathologist who was unaware of the preoperative MRI findings. Diseases extending into but not through the prostatic capsule were considered negative for ECE in pathological examination. Considering the effect on final pathological outcomes, patients who had undergone prior hormone treatment, radiotherapy or any ablative technique were excluded from this study.

All patients who had undergone RALP in our institution were registered in a specific database that includes all important information pertaining to tumour and patient characteristics, including age, prostate weight, initial prostate-specific antigen (PSA), number of biopsy cores, biopsy Gleason score, interval from biopsy to MRI evaluation, interpretation of MRI and pathological report. Following approval by the local institutional review board, data from enrolled patients were evaluated and analysed. Clinical and pathological stage were assigned according to the 1992 TNM staging system in this series based on digital rectal examination, transrectal ultrasound findings and MRI.

\section{Protocol for pelvic MRI and image interpretation}

MRI was performed using a 3.0-T whole-body MR scanner (Magnetom Tim Trio; Siemens Medical Systems, Erlangen, Germany) with a pelvic array coil (3T Body Matrix TIM Coil; Siemens Medical Systems). All MRI examinations were performed according to the same protocol. The imaging protocol included transverse T2-weighted turbo spin-echo sequence imaging, transverse unenhanced T1-weighted imaging and transverse echo planar diffusion-weighted imaging. The imaging parameters are summarized in Table 1. MR images were interpreted by a single experienced uroradiologist who is board-certified in both radiology and urology. The radiologist was aware that the patients had prostate cancer but was

Table 1 MRI sequence protocol

\begin{tabular}{lccc}
\hline Parameters & $\begin{array}{c}\text { T2-weighted } \\
\text { SE sequence }\end{array}$ & $\begin{array}{c}\text { T1-weighted } \\
\text { SE sequence }\end{array}$ & $\begin{array}{c}\text { Diffusion-weighted } \\
\text { Imaging }\end{array}$ \\
\hline Time (ms) & & & \\
$\quad$ Repetition & 3290 & 440 & 3150 \\
$\quad$ Echo & 120 & 11 & 71 \\
Matrix & $384 \times 384$ & $448 \times 359$ & $128 \times 104$ \\
Field of view (mm) & 250 & 250 & 280 \\
Section thickness (mm) & 4 & 4 & 4 \\
Flip angle $\left({ }^{\circ}\right)$ & 140 & 150 & - \\
b value $\left(\mathrm{s} \mathrm{mm}^{-2}\right)$ & - & - & 1000 \\
\hline
\end{tabular}

Abbreviations: MRI, magnetic resonance imaging; SE, spin echo. unaware of all other clinical and pathological findings, including Gleason score, tumour location at biopsy, clinical stage and outcome of digital rectal examination. Haemorrhage was considered present when an area of high signal intensity within the prostate was observed on T1-weighted MRI. The following criteria were used for diagnosis of ECE: tumour tissue in the extraprostatic tissue; obliteration of the rectoprostatic angle; bulging of the prostate contour caused by the tumour; asymmetry or direct involvement of the neurovascular bundles; thickening, retraction or irregularity of the prostate capsule; disruption of the prostatic capsule adjacent to the tumour; and stranding of the periprostatic fatty tissue. ${ }^{11}$ Capsular irregularity was defined as spiculated or streaky low signal intensity extending from the capsule, low signal intensity in the periprostatic tissue, or focal irregular bulging of the capsule. ${ }^{12}$ The criterion for SVI was abnormal asymmetric low signal intensity within the lumen or dilatation with or without asymmetry of the seminal vesicles on T2-weighted images. ${ }^{13}$

The primary end point of this series was the accuracy (matching of the pathological finding to the MRI-based prediction) between preoperative MRI and pathological outcome (Figure 1). Staging, and prediction of ECE and SVI were evaluated. SPSS (version 12.0 for Windows; SPSS Inc., Chicago, IL, USA) was used as a statistical program. Receiver operating characteristic (ROC) derived areas under the curve (AUCs) were estimated in order to evaluate the predictability of each clinical variable with regard to accuracy. Mann-Whitney $U$ test and logistic regression was used, and the exact method used is described in result session. Statistical significance in this study was set at $P<0.05$, and all reported $P$ values are two-sided.

\section{RESULTS}

The characteristics of the patients are summarized in Table 2. In $53.7 \%$ of patients, MRI was conducted within 3 weeks after biopsy, and intraprostatic haemorrhage was found in 87 images (71.9\%). After RALP, $43.8 \%$ (53/121) of the patients were matched with the MRI predicted stage. Upstaging from the T1c stage, which contains upstaging above T2a, was found in 48 patients (39.7\%), and no preoperative radiological tumour focus was found in nine patients (7.4\%). In these nine patients, although the mean Gleason score $(6.2 \pm 0.4)$ and mean initial PSA $\left(6.3 \pm 1.7 \mathrm{ng} \mathrm{ml}^{-1}\right)$ were lower than those of the patient with preoperative radiological tumour focus, the difference was not statistically significant $(P=0.15$ and 0.21 , respectively, by MannWhitney $U$ test). The pathological stage was lower than the MRI-predicted stage in 11 patients $(9.1 \%)$.

The estimated accuracy of MRI imaging was $69.4 \%$ for ECE, $84.3 \%$ for SVI and $73.6 \%$ for pathological stage T3 (Table 3). A false negative occurred in $51.4 \%$ and $52.6 \%$ for ECE and SVI, respectively.

When comparing the matched group with MRI findings in prediction of ECE, the unmatched group had significantly higher initial PSA $\left(12.8 \pm 11.0\right.$ versus $\left.8.1 \pm 4.6 \mathrm{ng} \mathrm{ml}^{-1}\right)$ in both univariate and multivariate logistic regression analysis ( $P=0.006$ and 0.048 ; Table 4$)$. In the prediction of SVI, initial PSA $\left(8.1 \pm 4.7\right.$ versus $17.3 \pm 13.2 \mathrm{ng} \mathrm{ml}^{-1}$, $P<0.001$ and 0.009$)$ and biopsy Gleason score $(6.5 \pm 1.0$ versus $7.6 \pm 1.2, P<0.001$ and 0.035 ) were significantly higher in the unmatched group than in the matched group (Table 5). However, in a comparison of the false-positive and -negative subgroups, no variable showed a statistical difference in prediction of either ECE or SVI (data not shown).

In ROC analysis, the AUC of initial PSA in accurate prediction of ECE was $0.652(P=0.008,95 \%$ confidence interval $(C I): 0.543-0.761$; Figure 2a). The AUCs of initial PSA and biopsy Gleason score in accurate prediction of SVI were $0.771(P<0.001,95 \%$ CI: $0.645-$ $0.897)$ and $0.748(P=0.001,95 \%$ CI: $0.626-0.870$, Figure $2 b)$, 

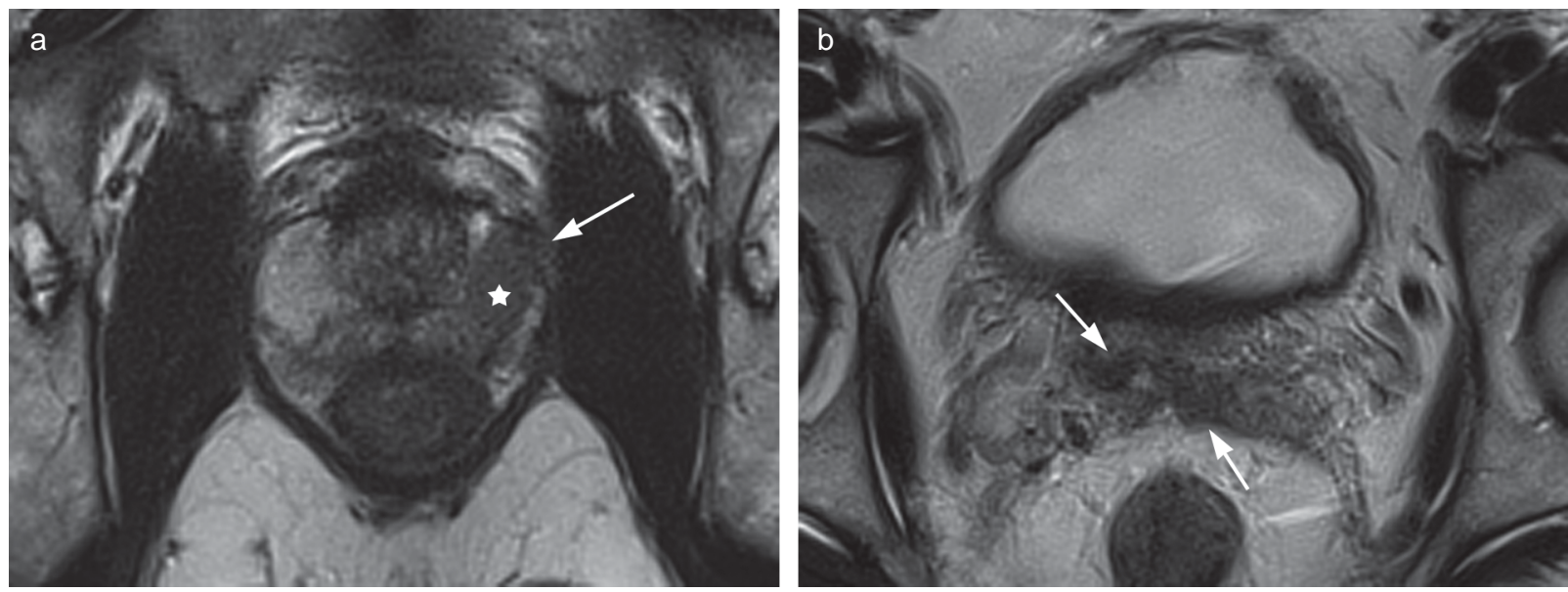

Figure 1 False-negative findings in preoperative MRI. (a) Transverse T2-weighted MRI shows an area of low signal intensity (asterisk) in the left peripheral zone with irregular bulging and disruption of the prostatic capsule (arrow), which are signs of locally advanced disease. Histopathological examination of the radical prostatectomy specimen confirmed that the tumour was confined within the prostatic capsule with surgical margins negative for tumour. (b) Transverse T2-weighted MRI shows areas of low signal intensity (arrows) in the medial aspect of the seminal vesicles displaying a sign of seminal vesicle invasion. Histopathological examination of the radical prostatectomy specimen confirmed only haemorrhage and inflammation in the seminal vesicles. MRI, magnetic resonance imaging.

respectively. Using a PSA cutoff of $10.33 \mathrm{ng} \mathrm{ml}^{-1}$, the sensitivity was $73.5 \%$ and the specificity was $63.2 \%$. With a Gleason score cutoff of 7.5 , the sensitivity was $83.3 \%$, and the specificity was $52.6 \%$. When applying clinical cutoffs of initial PSA of 10 and $20 \mathrm{ng} \mathrm{ml}^{-1}$, the accuracy of MRI in the prediction of ECE was significantly decreased

\section{Table 2 Patient characteristics}

\begin{tabular}{|c|c|c|}
\hline & Mean (range) & s.d. \\
\hline Age (year) & $62.8(46-74)$ & 6.3 \\
\hline Prostate weight (g) & $29.5(12-75)$ & 11.1 \\
\hline Initial PSA (ng ml $\left.{ }^{-1}\right)$ & $9.6(0.4-24.4)$ & 7.5 \\
\hline Number of biopsy core & $10.6(6-14)$ & 2.2 \\
\hline Biopsy Gleason score & $6.7(4-10)$ & 1.1 \\
\hline \multicolumn{3}{|l|}{ Risk stratification, $n(\%)$} \\
\hline Low risk & $50(41.3)$ & - \\
\hline Intermediate risk & $41(33.9)$ & - \\
\hline High risk & $30(24.8)$ & - \\
\hline \multicolumn{3}{|l|}{ Clinical stage, $n(\%)$} \\
\hline $\mathrm{T} 1$ & $9(7.4)$ & \\
\hline $\mathrm{T} 2$ & $93(76.9)$ & \\
\hline T2a & $30(24.8)$ & \\
\hline $\mathrm{T} 2 \mathrm{~b}$ & $21(17.4)$ & \\
\hline $\mathrm{T} 2 \mathrm{c}$ & $42(34.7)$ & \\
\hline T3 & $17(14.0)$ & \\
\hline ТЗа & $10(8.3)$ & \\
\hline T3b & $2(1.7)$ & \\
\hline T3c & $5(4.1)$ & \\
\hline T4 & $2(1.7)$ & \\
\hline \multicolumn{3}{|l|}{ Pathological stage, $n(\%)$} \\
\hline $\mathrm{T} 2$ & $78(64.5)$ & \\
\hline Т2а & $17(14.0)$ & \\
\hline $\mathrm{T} 2 \mathrm{~b}$ & $11(9.1)$ & \\
\hline $\mathrm{T} 2 \mathrm{c}$ & $50(41.3)$ & \\
\hline T3 & $38(31.4)$ & \\
\hline ТЗа & $21(17.4)$ & \\
\hline $\mathrm{T} 3 \mathrm{~b}$ & $7(5.8)$ & \\
\hline T3c & $10(8.3)$ & \\
\hline $\mathrm{T} 4$ & $5(4.1)$ & \\
\hline Interval after biopsy to MRI (day) & $26.0(2-110)$ & 17.2 \\
\hline
\end{tabular}

Abbreviations: MRI, magnetic resonance imaging; PSA, prostate-specific antigen. in the group with PSA over $20 \mathrm{ng} \mathrm{ml}^{-1}$ (75.6\%, 64.5\% and 37.5\%, $P=0.01$ by Chi-square test, Table 6), and this group also had significantly decreased accuracy of MRI in the prediction of SVI $(91.5 \%$, $77.4 \%$ and $37.5 \%, P<0.01$; Table 7). Applying the clinical cutoff of a Gleason score of 7, the accuracy of MRI in prediction of SVI was significantly decreased in the higher Gleason score group (93.9\%, $82.1 \%$ and $62.9 \%, P=0.01)$.

\section{DISCUSSION}

Treatment of prostate cancer is greatly dependent on local staging, and knowledge of the spread of cancer beyond the prostate capsule is critical for the selection of appropriate therapy. For clinically localized organ-confined disease, radical prostatectomy has been established as the standard treatment, providing a 10 -year progression-free probability in approximately $90 \%$ of men, ${ }^{14}$ whereas for patients with more advanced disease with the presence of ECE or SVI, surgery may carry a substantial risk of positive surgical margins, subsequently compromising cancer control. In addition, invasion of seminal vesicles markedly reduces long-term survival in patients undergoing radical prostatectomy. ${ }^{15}$ Thus, accurate preoperative evaluation of the local stage is pivotal in the selection of a proper modality for each patient.

Table 3 The accuracy of staging MRI in predicting pathological T3 disease

\begin{tabular}{|c|c|c|c|}
\hline & $\begin{array}{l}\text { Predict } \\
\text { extracapsular } \\
\text { extension }\end{array}$ & $\begin{array}{c}\text { Predict } \\
\text { seminal vesicle } \\
\text { invasion }\end{array}$ & $\begin{array}{c}\text { Predict } \\
\text { pathological } \\
\text { stage T3 }\end{array}$ \\
\hline Matched with final pathology, $n(\%)$ & $84(69.4)$ & $102(84.3)$ & $89(73.6)$ \\
\hline $\begin{array}{l}\text { Unmatched with final pathology, } n \\
(\%)\end{array}$ & $37(30.6)$ & $19(15.7)$ & $32(26.4)$ \\
\hline \multicolumn{4}{|l|}{ Categories for unmatching, $n(\%)$} \\
\hline $\begin{array}{l}\text { MRI predicted no lesion, but } \\
\text { pathology revealed the presence } \\
\text { of a lesion (false negativity in MRI) }\end{array}$ & $19(51.4)$ & $10(52.6)$ & \\
\hline $\begin{array}{l}\text { MRI predicted the presence of a } \\
\text { lesion, but pathology revealed } \\
\text { no lesion (false positivity in MRI) }\end{array}$ & $18(48.6)$ & $9(47.4)$ & \\
\hline
\end{tabular}

Abbreviation: MRI, magnetic resonance imaging. 
Table 4 The effects of clinical variables on the predictability of extracapsular extension in staging MRI

\begin{tabular}{|c|c|c|c|c|c|c|c|}
\hline \multirow{2}{*}{ Patients' characteristics } & \multirow{2}{*}{$\begin{array}{l}\text { Total patients } \\
(\mathrm{n}=121)\end{array}$} & \multicolumn{2}{|c|}{ Predictability in extracapsular extension } & \multicolumn{2}{|c|}{ Univariate } & \multicolumn{2}{|c|}{ Multivariate } \\
\hline & & $\begin{array}{l}\text { Matched group } \\
(\mathrm{n}=84,69.4 \%)\end{array}$ & $\begin{array}{l}\text { Unmatched group } \\
(\mathrm{n}=37,30.6 \%)\end{array}$ & $\mathrm{P}$ value & $\begin{array}{c}\text { Relative risk } \\
(95 \% \mathrm{Cl})\end{array}$ & $\mathrm{P}$ value & $\begin{array}{c}\text { Relative risk } \\
(95 \% \mathrm{CI})\end{array}$ \\
\hline Prostate weight (g) & $29.5 \pm 11.1$ & $29.9 \pm 10.8$ & $28.4 \pm 11.7$ & 0.46 & $0.99(0.95-1.02)$ & 0.74 & $0.99(0.95-1.04)$ \\
\hline Initial PSA (ng ml ${ }^{-1}$ ) & $9.6 \pm 7.5$ & $8.1 \pm 4.6$ & $12.8 \pm 11.0$ & 0.006 & $1.09(1.03-1.17)$ & 0.048 & $1.08(1.02-1.17)$ \\
\hline No. of biopsy cores & $10.6 \pm 2.2$ & $10.4 \pm 2.4$ & $11.0 \pm 1.5$ & 0.22 & $1.13(0.93-1.38)$ & 0.19 & $1.18(0.93-1.49)$ \\
\hline \multicolumn{8}{|l|}{ Clinical stage, $n(\%)$} \\
\hline $\mathrm{T} 1$ & $9(7.4)$ & $8(9.5)$ & $1(2.7)$ & - & - & - & - \\
\hline T2 & 93 (76.9) & $69(82.1)$ & $24(64.9)$ & 0.35 & $2.8(0.33-23.4)$ & 0.48 & $2.23(0.25-20.1)$ \\
\hline T3 & $17(14.0)$ & $7(8.3)$ & $10(27.0)$ & 0.04 & $11.4(1.15-113.1)$ & 0.09 & $7.8(0.72-85.50)$ \\
\hline T4 & $2(1.7)$ & - & $2(5.4)$ & - & - & - & - \\
\hline \multicolumn{8}{|l|}{ Risk stratification, $n(\%)$} \\
\hline No haemorrhage & $34(28.1)$ & $23(27.4)$ & $11(29.7)$ & 0.79 & $1.12(0.48-2.63)$ & 0.87 & $0.91(0.31-2.73)$ \\
\hline
\end{tabular}

Abbreviations: $\mathrm{Cl}$, confidence interval; MRI, magnetic resonance imaging; PSA, prostate-specific antigen.

Table 5 The effects of clinical variables on the predictability of seminal vesicle invasion in staging MRI

\begin{tabular}{|c|c|c|c|c|c|c|c|}
\hline \multirow[b]{2}{*}{ Patients' characteristics } & \multirow{2}{*}{$\begin{array}{l}\text { Total patients } \\
\quad(\mathrm{n}=121)\end{array}$} & \multicolumn{2}{|c|}{ Predictability in Seminal vesicle invasion } & \multicolumn{2}{|c|}{ Univariate } & \multicolumn{2}{|c|}{ Multivariate } \\
\hline & & $\begin{array}{c}\text { Matched group } \\
(\mathrm{n}=102,84.3 \%)\end{array}$ & $\begin{array}{c}\text { Unmatched group } \\
(\mathrm{n}=19,15.7 \%)\end{array}$ & $\mathrm{P}$ value & $\begin{array}{c}\text { Relative risk } \\
(95 \% \mathrm{Cl})\end{array}$ & $\mathrm{P}$ value & $\begin{array}{l}\text { Relative risk (95\% } \\
\text { Cl) }\end{array}$ \\
\hline Prostate weight (g) & $29.5 \pm 11.1$ & $30.3 \pm 11.6$ & $25.3 \pm 6.7$ & 0.074 & $0.95(0.89-1.005)$ & 0.11 & $0.92(0.84-1.02)$ \\
\hline Initial PSA (ng ml ${ }^{-1}$ ) & $9.6 \pm 7.5$ & $8.1 \pm 4.7$ & $17.3 \pm 13.2$ & $<0.001$ & $1.16(1.07-1.26)$ & 0.009 & $1.19(1.05-1.36)$ \\
\hline No. of biopsy core & $10.6 \pm 2.2$ & $10.6 \pm 2.3$ & $10.9 \pm 1.6$ & 0.52 & $1.08(0.85-1.39)$ & 0.53 & $1.13(0.78-1.62)$ \\
\hline \multicolumn{8}{|l|}{ Clinical stage, $n(\%)$} \\
\hline $\mathrm{T} 1$ & $9(7.4)$ & $9(8.8)$ & - & - & - & - & - \\
\hline $\mathrm{T} 2$ & $93(76.9)$ & $82(80.4)$ & $11(57.9)$ & 0.28 & $0.87(0.83-13.7)$ & 0.36 & $0.66(0.70-11.2)$ \\
\hline T3 & $17(14.0)$ & $10(9.8)$ & $7(36.8)$ & 0.01 & $4.75(1.15-98.1)$ & 0.07 & $3.21(0.92-68.5)$ \\
\hline T4 & $2(1.7)$ & $1(1.0)$ & $1(5.3)$ & 0.72 & $6.82(2.42-5.21)$ & 0.99 & $4.78(1.87-3.93)$ \\
\hline \multicolumn{8}{|l|}{ Risk stratification, $n(\%)$} \\
\hline No haemorrhage & $34(28.1)$ & $29(28.4)$ & $5(26.3)$ & 0.85 & $0.90(0.30-2.72)$ & 0.23 & $0.28(0.04-2.24)$ \\
\hline
\end{tabular}

Abbreviations: $\mathrm{Cl}$, confidence interval; MRI, magnetic resonance imaging; PSA, prostate-specific antigen.

Currently, MRI is believed to play an important role in the local staging of prostate cancer, and many centres in the United States are now routinely performing MRI and MR spectroscopic imaging of the prostate, mainly due to the ability of MRI to generate high-resolution anatomical images based on various inherent tissue characteristics. In addition, MRI also enables functional assessment using techniques such as diffusion-weighted MRI, MR spectroscopy and dynamic contrast-enhanced MRI (DCE-MRI).

The peculiarity of this series is that our data showed that radiological accuracy was affected by clinical variables. Predictability of ECE was affected by initial PSA, and predictability of SVI was affected by initial PSA and biopsy Gleason score. Of particular interest is that the initial PSA and Gleason scores were higher in the mismatched group compared with the matched counterpart. While a clinically acceptable cutoff was not determined, the AUC in ROC using initial PSA was 0.652 in the prediction of ECE and 0.771 in the prediction of SVI. Using the clinical cutoffs of PSA 10 and $20 \mathrm{ng} \mathrm{ml}^{-1}$ and a Gleason score of 7 points, accuracy decreased significantly in the high PSA and Gleason score groups. These observations imply that for patients with PSA over $20 \mathrm{ng} \mathrm{ml}^{-1}$ or Gleason score over 7 points, the presence of ECE or SVI in specific sites on the prostate cannot be reliably predicted with only preoperative MRI findings that were conducted after prostate biopsy.

However, this finding is opposite to that of other researchers. Ellis et al. ${ }^{16}$ reported that high-grade tumours are more likely to be detected on a T2 sequence. Later, Ikonen et al. ${ }^{17}$ confirmed that endorectal MRI can detect poorly differentiated prostate cancer lesions more 

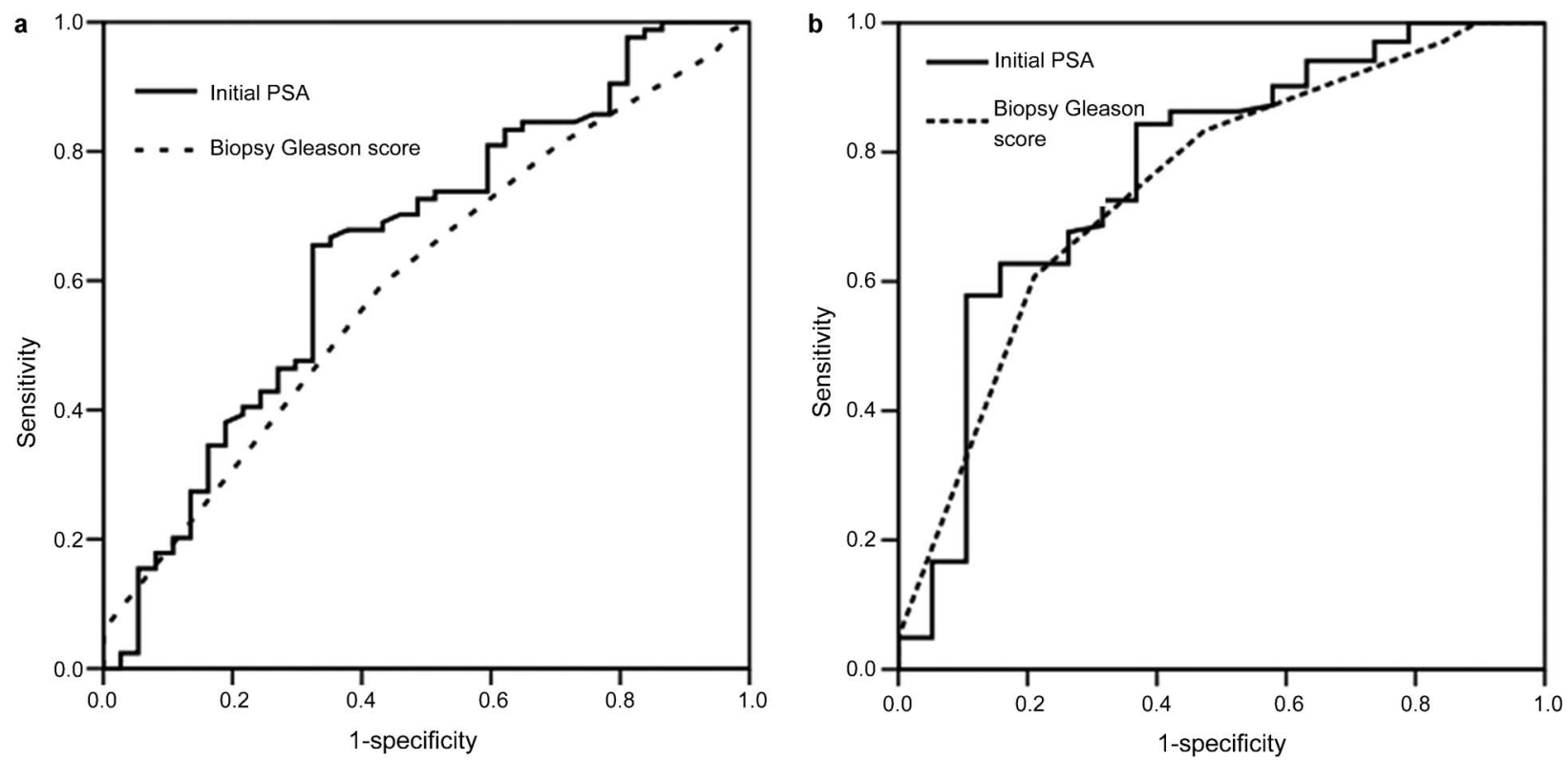

Figure 2 ROC analysis of initial PSA and Gleason scores in the accurate prediction of (a) extracapsular extension and (b) seminal vesicle invasion. ROC, receiver operating characteristic; PSA, prostate-specific antigen.

Table 6 Discrepancy in predicting ECE based on initial PSA

\begin{tabular}{|c|c|c|c|c|c|c|}
\hline & \multirow{2}{*}{ No. of patients (\%) } & \multicolumn{5}{|c|}{ No. of ECE cases/total No. (\%) } \\
\hline & & Sensitivity & Specificity & $P P V$ & $N P V$ & Accuracy \\
\hline Overall & 121 & 9/27 (33.3) & 76/94 (80.9) & 9/27 (33.3) & 76/94 (80.9) & $85 / 121(70.2)$ \\
\hline$<10$ & $82(67.8)$ & 2/9 (22.2) & $60 / 73(82.2)$ & 2/15 (13.3) & 60/67 (89.6) & $62 / 82(75.6)^{*}$ \\
\hline 10-20 & 31 (25.6) & 5/13 (38.5) & 15/18 (83.3) & $5 / 8(62.5)$ & 15/23 (65.2) & 20/31 (64.5)* \\
\hline$>20$ & $8(6.6)$ & $2 / 5(40.0)$ & 1/3 (33.3) & $2 / 4(50.0)$ & $1 / 4(25.0)$ & $3 / 8(37.5) *$ \\
\hline
\end{tabular}

Abbreviations: ECE, extracapsular extension; NPV, negative predictive value; PPV, positive predictive value; PSA, prostate-specific antigen.

$* P=0.01$, by Chi-square test.

Table 7 Discrepancy in predicting SVI based on Gleason score and initial PSA

\begin{tabular}{|c|c|c|c|c|c|c|}
\hline & No. of patients (\%) & \multicolumn{5}{|c|}{ No. of SVI cases/total No. (\%) } \\
\hline Overall & 121 & 2/12 (16.7) & $100 / 109$ (91.7) & 2/11 (18.2) & 100/110 (90.9) & 102/121 (84.3) \\
\hline$<7$ & $66(54.5)$ & $0 / 1(0)$ & $62 / 65(95.4)$ & $0 / 3(0)$ & $62 / 63(98.4)$ & $62 / 66(93.9)^{*}$ \\
\hline 7 & $28(23.1)$ & $0 / 3(0)$ & 23/25 (92) & $0 / 2(0)$ & 23/26 (88.5) & $23 / 28(82.1)^{*}$ \\
\hline$>7$ & $27(22.3)$ & $2 / 8(25.0)$ & $15 / 19(78.9)$ & 2/6 (33.3) & $15 / 21(71.4)$ & $17 / 27(62.9) *$ \\
\hline $10-20$ & $31(25.6)$ & $2 / 8(25.0)$ & 22/23 (95.7) & $2 / 3(66.7)$ & 22/28 (78.6) & $24 / 31(77.4)^{* *}$ \\
\hline$>20$ & $8(6.6)$ & $0 / 1(0)$ & $3 / 7(42.9)$ & $0 / 4(0)$ & $3 / 4(75.0)$ & $3 / 8(37.5) * *$ \\
\hline
\end{tabular}

Abbreviations: NPV, negative predictive value; PPV, positive predictive value; PSA, prostate-specific antigen; SVI, seminal vesicle invasion.

$* P=0.01, * * P<0.01$, by Chi-square test.

accurately than well-differentiated tumours, although there was no statistically significant difference between PSA groups in detection of tumours. In a recent series using DCE-MRI, the sensitivity and specificity for identification of $>10 \%$ of Gleason grade $4 / 5$ were 81 and $82 \%$, respectively. ${ }^{18}$ These observations, which were in discordance with ours, require explanation here. We hypothesize the characteristics of our data, which show a relatively short interval from prostate biopsy to conducting preoperative MRI, and evaluating ECE or SVI instead of identification of intraprostatic lesion.

In this series, we evaluated the accuracy of MRI using pathological outcome as a reference. Each assessed MRI method showed good specificity and positive predictable value but low sensitivity and negative predictable value in the detection of characteristics of prostate cancer. The use of MRI in the post-biopsy setting is associated with 
a significant artefact that confounds MRI accuracy. In MRI, T2weighted imaging provides high-resolution morphological imaging of the gland in the three planes, and axial T1-weighted imaging is used in detection of post-biopsy haemorrhage. While cancer has generally been identified as a low signal in T2-weighted imaging, there are numerous false positives (inflammation, scars, post-radiotherapy appearance, etc.), and infiltrating cancers may not show such a typical appearance. Among these factors, post-biopsy haemorrhage represents the most common obstacle preventing accurate detection of prostate cancer because most patients undergo imaging of the prostate after biopsy. A haemorrhage resulting from a biopsy can show areas of low signal intensity that are similar to those of prostate cancer, and thus the haemorrhage causes discrepancies between the results of MRI and histopathology. ${ }^{19}$ Post-biopsy T1-weighted MRI has been shown to exhibit high signal-intensity changes in $28 \%-77 \%$ of patients. ${ }^{5,20}$ Due to these limitations, investigators in prior studies have recommended an interval of 3 weeks between biopsy and MRI, ${ }^{20}$ and investigators in a more recent study suggested that a post-biopsy interval of 8 weeks before MRI may be more beneficial. ${ }^{12}$ However, in this series, the mean interval from biopsy to MRI was only 26 days, and MRI was taken within 3 weeks in $53.7 \%$ of patients. In this series, haemorrhage was found in $71 \%$ of patients, and the presence of intraprostatic haemorrhage may have had a negative effect on the predictive ability of MRI. To overcome the artefacts mainly induced by post-biopsy haemorrhage, some researchers suggested conducting MRI before prostate biopsy. Using DCE-MRI, Hara et al. ${ }^{21}$ demonstrated a sensitivity of $93 \%$ and a specificity of $96 \%$ in 90 men with elevated PSA who had MRI before prostate biopsy. However, voxels suspicious of malignancy were demonstrated in 44 patients, with only 11 of these having cancer on biopsy, hence leading to a specificity of $54 \%$ and an accuracy of $60 \%$. While MRI applied in a pre-biopsy setting may potentially increase the predictability of MRI, more data will be needed to obtain actual evidence supporting this strategy.

Studies using whole-mount histology as the reference standard have reported T2-weighted MRI scans with broad spectrums of sensitivity ranging between $37 \%$ and $96 \%{ }^{22}$ The wide variation in results is a consequence of equipment, patient selection, experience and the accuracy of pathological correlation. ${ }^{23}$ In addition, interobserver variability for the reference of each finding is also likely to affect the outcome. ${ }^{24}$ For example, ECE can be detected on T2-weighted images by visualisation of the direct extension of the tumour into the periprostatic fat or by an interruption in the usually continuous black line representing the prostate capsule. Capsular irregularity is also considered an important MRI sign of ECE; ${ }^{9,25}$ however, this irregularity may be related to trauma, including prostatic biopsy. ${ }^{5}$ Actually, more than 20 different criteria exist for ECE. ${ }^{26}$ In fact, previous studies have shown that if a diagnosis of extraprostatic tumour spread is based solely on obvious findings, the high specificity may be associated with a downside of extremely poor sensitivity. ${ }^{27}$ When stricter definitions were used, accuracy rates fell from an average sensitivity of 70\%-80\% to below $40 \%$ in one particular report. ${ }^{28}$ Using contour deformity with only irregular margins as a criterion for capsular penetration, Chelsky et al. ${ }^{9}$ achieved an increase in specificity from $78 \%$ to $96 \%$. In addition, the degree of ECE was associated with the predictability of ECE by MRI. The sensitivity of endorectal T2-weighted-MRI for extracapsular extension of less than $1 \mathrm{~mm}$ was only $14 \%$ compared with $71 \%$ for extracapsular extension greater than $1 \mathrm{~mm}^{29}$

SVI, which shows low-signal foci within an otherwise high-signal chamber, can be directly visualized as extension of the tumour from the base of the prostate or as a low-signal intensity lesion that is discontinuous from the primary tumour. However, in elderly men, atrophic changes within the seminal vesicles can render the entire seminal vesicle low in signal on T2-weighted imaging, thus interfering with the diagnosis. False-positive findings of SVI can occur as a result of post biopsy haemorrhage or inflammatory changes. ${ }^{5,30}$

We recognize several limitations in this series. Because we did not have a policy regarding the timing of MRI after prostate biopsy, MRI was performed earlier than in other series. While this MRI interval was not a significant variable statistically affecting the accuracy of the pathological finding in this series, it is not certain whether the finding that predictability of ECE and SVI was negatively affected by the presence of higher initial PSA or Gleason score can be translated to other patient groups with a longer MRI interval. Additionally, we did not evaluate the degree of haemorrhage, mainly because we believe that there is no reliable tool for exact assessment of the degree. In addition, because this study was based on actual clinical data before radical prostatectomy in our institution, all MR images in this series could be interpreted only by a single full-faculty uroradiologist, as opposed to other research based on retrospective image interpretation by several radiologists. This limitation also hindered subjective estimation of the degree of haemorrhage. However, considering that degree of haemorrhage showed a significantly negative correlation with tumour size ${ }^{31}$ it is possible that a large haemorrhage from a small tumour, resulting in capsular irregularity, could be misinterpreted as ECE. Finally, the use of MRI in treatment of prostate cancer is evolving. Various functional MR images, including diffusion-weighted MRI, MRI, DCE-MRI and endorectal coil MRI, may improve cancer detection and localisation, as well as information regarding the biological behaviour, volume and staging of cancers for individualized therapy. ${ }^{32}$ However, each technique has one or more limitations, such as no standard parameters or low accuracy in central region of the gland. ${ }^{33}$ Particularly for endorectal coil MRI, while it had been reported to have advanced sensitivity and specificity for detecting T3 disease over $90 \%$, controversy still exists over the role of endorectal coil MRI of the prostate in detecting locally advanced disease, ${ }^{34,35}$ with unique complications including rectal bleeding, erosion and proctitis. Considering these limitations and cost effectiveness, these functional images and endorectal coil MRI were not routinely conducted in our institution. We think that a comprehensive understanding of the advantages and disadvantages of various MR imaging techniques and protocols will improve the MR-based detection and localisation of prostate cancer.

In summary, staging MRI after prostate biopsy demonstrated modest accuracy in the prediction of ECE and SVI using pathological outcome as a reference in patient groups with no previously set period for MRI evaluation after initial biopsy. The presence of higher initial PSA or Gleason score negatively affected the accuracy of the prediction of ECE and SVI. Thus, for these patient groups, to obtain margin negativity, operative findings rather than preoperative MR images may provide substantial information, implying a clinical advantage in conducting MRI in the pre-biopsy setting in selected patients.

\section{AUTHOR CONTRIBUTIONS}

YHK designed the study and drafted the paper. DJS provided initial radiological interpretation of the patient and radiological supervision on this paper. SGK performed basic statistic analysis. SHK conducted statistic analysis and acquisition of data. JGL contributed on acquisition of data. JJK is the editorial supervision on the draft. JC contributed on acquisition of data and supervision the design of the study. 


\section{COMPETING FINANCIAL INTERESTS}

The authors declare no competing financial interests.

1 Borley N, Feneley MR. Prostate cancer: diagnosis and staging. Asian J Androl 2009; 11: 74-80.

2 Wang L, Mullerad M, Chen HN, Eberhardt SC, Kattan MW et al. Prostate cancer:incremental value of endorectal MR imaging findings for prediction of extracapsular extension. Radiology 2004; 232: 133-9.

3 Wang L, Hricak H, Kattan MW, Schwartz LH, Eberhardt SC et al. Combined endorectal and phased-array MRI in the prediction of pelvic lymph node metastasis in prostate cancer. Am J Roentgenol 2006; 186: 743-8.

4 Wang L, Hricak H, Kattan MW, Chen H, Scardino PT. Prediction of prostate cance organ-confined disease: the incremental value of endorectal coil magnetic resonance imaging to partin staging nomograms (2001version). Radiology 2006; 238: $597-$ 603.

5 White S, Hricak H, Forstner R, Kurhanewicz J, Vigneron DB et al. Prostate cancer: effect of postbiopsy hemorrhage on interpretation of MR images. Radiology 1995; 195: 385-90.

6 Fuchsjäger M, Shukla-Dave A, Akin O, Barentsz J, Hricak H. Prostate cancer imaging. Acta Radiol 2008; 49: 107-20.

7 Ikonen S, Kivisaari L, Vehmas T, Tervahartiala P, Salo JO et al. Optimal timing of postbiopsy MR imaging of the prostate. Acta Radiol 2001; 42: 70-3.

8 Sommer FG, Nghiem HV, Herfkens R, McNeal J, Low RN. Determining the volume of prostatic carcinoma: value of MR imaging with an external-array coil. $A J R A m ~ J$ Roentgenol 1993; 161: 81-6.

9 Chelsky MJ, Schnall MD, Seidmon EJ, Pollack HM. Use of endorectal surface coil magnetic resonance imaging for local staging of prostate cancer. J Urol 1993; 150 391-5.

10 Gronau E, Goppelt M, Harzmann R, Weckermann D. Prostate cancer relapse after therapy with curative intention: a diagnostic and therapeutic dilemma. Onkologie 2005; 28: 361-6.

11 Fütterer JJ. MR imaging in local staging of prostate cancer. Eur J Radio/ 2007; 63 328-34.

12 Qayyum A, Coakley FV, Lu Y, Olpin JD, Wu L et al. Organ-confined prostate cancer: effect of prior transrectal biopsy on endorectal MRI and MR spectroscopic imaging. AJR Am J Roentgenol 2004; 183: 1079-83.

13 Rørvik J, Halvorsen OJ, Albrektsen G, Ersland L, Daehlin L et al. MRI with an endorectal coil for staging of clinically localised prostate cancer prior to radical prostatectomy. Eur Radiol 1999; 9: 29-34.

14 Payne H. Management of locally advanced prostate cancer. Asian J Androl 2009; 11 81-7.

15 Epstein JI, Amin M, Boccon-Gibod L, Egevad L, Humphrey PA et al. Prognostic factors and reporting of prostate carcinoma in radical prostatectomy and pelvic lymphadenectomy specimens. Scand J Urol Nephrol Supp/ 2005; 216: 34-63.

16 Ellis JH, Tempany C, Sarin MS, Gatsonis C, Rifkin MD et al. MR imaging and sonography of early prostatic cancer: pathologic and imaging features that influence identification and diagnosis. AJR Am J Roentgenol 1994; 162: 865-72.
17 Ikonen S, Kärkkäinen P, Kivisaari L, Salo JO, Taari K et al. Magnetic resonance imaging of prostatic cancer: does detection vary between high and low Gleason score tumors? Prostate 2000; 43: 43-8.

18 Puech P, Potiron E, Lemaitre L, Leroy X, Haber GP et al. Dynamic contrast-enhancedmagnetic resonance imaging evaluation of intraprostatic prostate cancer: correlation with radical prostatectomy specimens. Urology 2009; 74: 1094-9.

19 Lee HW, Seo SI, Jeon SS, Lee HM, Choi HY. Can we predict real T3 stage prostate cancer in patients with clinical T3 (cT3) disease before radical prostatectomy? Yonsei Med J 2010; 51: 700-7.

20 Ikonen S, Kivisaari L, Vehmas T, Tervahartiala P, Salo JO et al. Optimal timing of postbiopsy MR imaging of the prostate. Acta Radiol 2001; 42: 70-3.

21 Hara N, Okuizumi M, Koike H, Kawaguchi M, Bilim V. Dynamic contrast-enhanced magnetic resonance imaging (DCE-MRI) is a useful modality for the precise detection and staging of early prostate cancer. Prostate 2005; 62: 140-7.

22 Ahmed HU, Kirkham A, Arya M, Illing R, Freeman A et al. Is it time to consider a role for MRI before prostate biopsy? Nat Rev Clin Oncol 2009; 6: 197-206.

23 Oehr P, Bouchelouche K. Imaging of prostate cancer. Curr Opin Oncol2007; 19: 259 64

24 Carey BM. Imaging for prostate cancer. Clin Oncol 2005; 17: 553-9.

25 Kurhanewicz J, Vigneron DB, Males RG, Swanson MG, Yu KK et al. The prostate gland: a clinically relevant approach to imaging. Radiol Clin North Am 2000; 38: 116-38.

26 Engelbrecht MR, Jager GJ, Laheij RJ, Verbeek AL, van Lier HJ et al. Local staging of prostate cancer using magnetic resonance imaging: a meta-analysis. Eur Radiol 2002; 12: 2294-302.

27 Outwater EK, Petersen RO, Siegelman ES, Gomella LG, Chernesky CE et al. Prostate carcinoma: assessment of diagnostic criteria for capsular penetration on endorectal coil MR images. Radiology 1994; 193: 333-9.

28 Dhingsa R, Qayyum A, Coakley FV, Lu Y, Jones KD et al. Prostate cancer localization with endorectal MR imaging and MR spectroscopic imaging: effect of clinical data on reader accuracy. Radiology 2004; 230: 215-20.

29 Jager GJ, Ruijter ET, van de Kaa CA, de la Rosette JJ, Oosterhof GO et al. Local staging of prostate cancer with endorectal MR imaging: correlation with histopathology. AJR Am J Roentgenol 1996; 166: 845-52.

30 Mirowitz SA. Seminal vesicles: biopsy-related hemorrhage simulating tumor invasion at endorectal MR imaging. Radiology 1992; 185: 373-6.

31 Tamada T, Sone T, Jo Y, Yamamoto A, Yamashita T et al. Prostate cancer: relationships between postbiopsy hemorrhage and tumor detectability at MR diagnosis. Radiology 2008; 248: 531-9.

32 Choi YJ, Kim JK, Kim N, Kim KW, Choi EK et al. Functional MR imaging of prostate cancer. Radiographics 2007; 27: 63-75.

33 Turkbey B, Albert PS, Kurdziel K, Choyke PL. Imaging localized prostate cancer: current approaches and new developments. AJR Am J Roentgenol 2009; 192 1471-80.

34 Lee SH, Park KK, Choi KH, Lim BJ, Kim JH et al. Is endorectal coil necessary for the staging of clinically localized prostate cancer? Comparison of non-endorectal versus endorectal MR imaging. World J Urol 2010; 28: 667-72.

35 Brajtbord JS, Lavery HJ, Nabizada-Pace F, Senaratne P, Samadi DB. Endorecta magnetic resonance imaging has limited clinical ability to preoperatively predict pT3 prostate cancer. BJU Int; e-pub ahead of print 26 August 2010; doi: 10.1111/ j.1464-410X.2010.09599.x. 